\title{
ON A CONVEXITY PROPERTY OF THE RANGE OF A MAXIMAL MONOTONE OPERATOR
}

\author{
JEAN-PIERRE GOSSEZ
}

\begin{abstract}
An example is given which shows that the closure of the range of a maximal monotone operator from a (nonreflexive) Banach space into its dual is not necessarily convex.
\end{abstract}

Introduction. Let $X$ be a real Banach space with dual $X^{*}$ and let $T: X$ $\rightarrow 2^{X^{*}}$ be a maximal monotone operator with domain $D(T)$ and range $R(T)$. In general $R(T)$ is not a convex set (cf. [4]) but it is known that when $X$ is reflexive, the (norm) closure of $R(T)$ is convex (cf. [5]). Without reflexivity, the convexity of $\mathrm{cl} R(T)$ is still true when $T$ is the subdifferential of a lower semicontinuous proper convex function (cf. [1]), or more generally, when the associated monotone operator $T_{1}: X^{* *} \rightarrow 2^{X^{*}}$ is maximal (cf. [2] where the proof is given under a slightly stronger assumption). Here $T_{1}$ denotes the operator whose graph is defined by

$$
\begin{aligned}
& \operatorname{gr} T_{1}=\left\{\left(x^{* *}, x^{*}\right) \in X^{* *} \times X^{*} ; \exists \text { a net }\left(x_{i}, x_{i}^{*}\right) \in \operatorname{gr} T\right. \text { with } \\
&\left.x_{i} \text { bounded, } x_{i} \rightarrow x^{* *} \text { weak }^{* *} \text { and } x_{i}^{*} \rightarrow x^{*} \text { in norm }\right\} .
\end{aligned}
$$

( $X$ is identified as usual to a subspace of its bidual $X^{* *}$.) The question was raised some years ago as to whether or not the convexity of $\mathrm{cl} R(T)$ holds in general.

In this note we answer this question negatively. We exhibit a (everywhere defined and coercive) maximal monotone operator from $l^{1}$ to $2^{10}$ whose range has not a convex closure. Our construction is based on a result of [3].

Example. Let $A: l^{1} \rightarrow l^{\infty}$ be the bounded linear operator defined by

$$
(A x)_{n}=\sum_{m=1}^{\infty} x_{m} \alpha_{m n}
$$

for $x=\left(x_{1}, x_{2}, \ldots\right) \in l^{1}$, where $\alpha_{m n}=0$ if $m=n, \alpha_{m n}=-1$ if $n>m$ and $\alpha_{m n}=+1$ if $n<m$. Let $J: l^{1} \rightarrow 2^{l \infty}$ be the usual duality mapping:

$$
J x=\left\{|x|_{l^{1}}\left(s\left(x_{1}\right), s\left(x_{2}\right), \ldots\right)\right\}
$$

where $s: \mathbf{R} \rightarrow 2^{\mathbf{R}}$ is given by $s(t)=-1$ if $t<0, s(t)=[-1,+1]$ if $t$ $=0$ and $s(t)=+1$ if $t>0$. For $\lambda>0$, the mapping $\lambda J+A$ is clearly max-

Received by the editors March 31, 1975.

AMS (MOS) subject classifications (1970). Primary 47H05; Secondary 46B10, $35 \mathrm{~J} 60$.

Key words and phrases. Nonreflexive Banach space, maximal monotone operator, convex set, bidual space.

(1) American Mathematical Society 1976 
imal monotone and coercive. It was shown in [3] that there exists $\lambda>0$ such that $R(\lambda J+A)$ is not dense in $l^{\infty}$ (for the norm topology of $l^{\infty}$ ).

Proposition. Let $\lambda>0$ be such that $R(\lambda J+A)$ is not dense in $l^{\infty}$. Then cl $R(\lambda J+A)$ is not a convex set.

Proof. Assume by contradiction that $\operatorname{cl} R(\lambda J+A)$ is convex. Since $y$ $\in \operatorname{cl} R(\lambda J+A)$ implies that the whole line $\{r y ; r \in \mathbf{R}\}$ is contained in cl $R(\lambda J+A), \mathrm{cl} R(\lambda J+A)$ would be a proper closed subspace of $l^{\infty}$. Consequently there would exist a nonzero $\mu \in\left(l^{\infty}\right)^{*}$ which vanishes on $R(\lambda J+A)$. We will show that this is impossible.

Let $\mu \in\left(l^{\infty}\right)^{*}$ vanish on $R(\lambda J+A)$. Denoting by $\beta \mathbf{N}$ the Stone-Čech compactification of $\mathbf{N}$, one can identify $l^{\infty}$ to the space $C(\beta \mathbf{N})$ of the continuous real-valued functions on $\beta \mathbf{N}$ and $\left(l^{\infty}\right)^{*}$ to the space $\Re(\beta \mathbf{N})$ of the Radon measures on $\beta \mathbf{N}$. We first show that $\mu_{i}=0$ for each $i \in \mathbf{N}$, where $\mu_{i}$ denotes the $\mu$-measure of $\{i\} \subset \beta \mathbf{N}$. The points

$$
y_{t}=(\lambda t, \lambda-1 / 2,-\lambda-1 / 2,0,0, \ldots),
$$

$t \in[-1,+1]$, belong to the image of $(0,1 / 2,-1 / 2,0,0, \ldots)$ by $(\lambda J+A)$. Thus $\left\langle\mu, y_{t}\right\rangle=0$ for all $t \in[-1,+1]$, which implies $\mu_{1}=0$. Considering the image of $(0,0,1 / 2,-1 / 2,0,0, \ldots)$ by $(\lambda J+A)$, one similarly gets $\mu_{2}=0$. And so on. We now prove that $\mu=0$, i.e. that $\langle\mu, y\rangle=0$ for all $y \in l^{\infty}$. Let $y$ $=\left(y_{1}, y_{2}, \ldots\right) \in l^{\infty}$. The image of $(k,-k, 0,0, \ldots), k>0$, by $(\lambda J+A)$ is

$$
\{(2 \lambda k-k,-2 \lambda k-k, 2 \lambda k s, 2 \lambda k t, \ldots) ; s, t, \ldots \in[-1,+1]\}
$$

thus, if $k$ is chosen sufficiently large, this image contains the point

$$
\tilde{y}=\left(2 \lambda k-k,-2 \lambda k-k, y_{3}, y_{4}, \ldots\right) \text {. }
$$

But $\tilde{y}_{j}=y_{j}$ for almost every $j \in \mathbf{N}$. Since $\mu_{i}=0$ for $i \in \mathbf{N}$, it follows that $\langle\mu, y\rangle=\langle\mu, \tilde{y}\rangle$, which is zero since $\tilde{y} \in R(\lambda J+A)$. Q.E.D.

\section{REFERENCES}

1. A. Brondsted and R. T. Rockafellar, On the subdifferentiability of convex functions, Proc. Amer. Math. Soc. 16 (1965), 605-611. MR 31 \#2361.

2. J.-P. Gossez, Opérateurs monotones non linéaires dans les espaces de Banach non réflexifs, J. Math. Anal. Appl. 34 (1971), 371-395. MR 47 \#2442.

3. - On the range of a coercive maximal monotone operator in a nonreflexive Banach space, Proc. Amer. Math. Soc. 35 (1972), 88-92. MR 45 \# 7544.

4. R. T. Rockafellar, Convex functions, monotone operators and variational inequalities, Theory and Applications of Monotone Operators (Proc. NATO Advanced Study Inst., Venice, 1968), Edizioni “Oderisi”, Gubbio, 1969, pp. 35-65. MR 41 \#6028.

5. - On the virtual convexity of the domain and range of a nonlinear monotone operator, Math. Ann. 185 (1970), 81 -90. MR 41 \#330.

Département de Mathematique, Université libre de Bruxelles, Avenue F. D. Roosevelt, 50, 1050 Bruxelles, BelgiQue 